\title{
Habituation in Stentor: A Response-Dependent Process
}

\author{
David C. Wood \\ Department of Behavioral Neuroscience, University of Pittsburgh, Pittsburgh, Pennsylvania 15260
}

\begin{abstract}
The contractile protozoan Stentor coeruleus habituates during repetition of the mechanical stimulus used to elicit the initial contractions. This decrement in response probability was found to be highly correlated with a reduction in receptor potential amplitude, while the amplitude of the action potentials that triggered the contractions did not change. When mechanical stimulation elicited receptor potentials and not action potentials, the receptor potential did not habituate significantly. Conversely, action potentials repetitively elicited by current pulses habituated animals to mechanical stimuli. Similarly, voltage steps used to simulate action potentials produced pronounced decrements in receptor currents recorded from voltage-clamped cells, while mechanical stimulation produced only small decrements. Thus, habituation depends primarily on action potential production, while mechanical stimulation itself makes a much smaller, but significant, contribution. The temporal relation between mechanical stimuli and action potentials, when both occur, is inconsequential in determining the rate and degree of habituation produced.
\end{abstract}

Habituation is defined as the decrement in response that occurs during the course of repeated stimulation. In a number of metazoan systems, habituation of a behavioral response has been traced to a decrement in function at identified synapses (Spencer et al., 1966; Castellucci et al., 1970; Zucker, 1972). In several cases, these decrements in synaptic function have been observed to result from progressive losses in transmitter release from presynaptic terminals (Castelluci and Kandel, 1974; Glanzman and Thompson, 1980). Modcls of the ccllular processes underlying these presynaptic changes assume either that synaptic vesicles are being released from an available pool more quickly than they are mobilized into it (e.g., Gingrich and Byrne, 1985) or that $\mathrm{Ca}^{2+}$ channels are being inactivated more quickly than they recover from inactivation (Klein et al., 1980). These models are similar in assuming that repetitive activation of a particular presynaptic process results in the depletion of a component necessary for that process. The data presented below indicate that depletion resulting from repetitive activation is inadequate as an explanation of habituation in Stentor. Instead, habituation in this organism depends on feedback from processes that are distinct from the process undergoing habituation.

Stentor is a large blue-green trumpet-shaped protozoan that

\footnotetext{
Received May 27, 1987; accepted Nov. 27, 1987.

I wish to acknowledge the helpful commentary of Dr. German Barrioneuvo during the preparation of this manuscript.

Correspondence should be addressed to Dr. David C. Wood, 410 Langley Hall, Department of Behavioral Neuroscience, University of Pittsburgh, Pittsburgh, PA 15260.

Copyright (c) 1988 Society for Neuroscience $0270-6474 / 88 / 072248-06 \$ 02.00 / 0$
}

contracts in an all-or-none fashion into a ball in response to suprathreshold mechanical, electrical, or photic stimuli. In the $30-45 \mathrm{sec}$ following a contraction, the animal generally reextends to the trumpet-shaped sessile form. If a mechanical stimulus is repetitively applied at $1 \mathrm{~min}$ intervals, the probability of eliciting a contraction decreases exponentially over the course of trials (Jennings, 1906). This decrement in response probability has been parametrically characterized and found to correspond to 7 of the 9 characteristics of habituation (Thompson and Spencer, 1966; Wood, 1970a, 1972, 1973). Dishabituation and habituation of dishabituation were not observed. Nevertheless, the response probability decrement observed in Stentor appears to be properly categorized as an example of habituation, since dishabituation has been shown to be dependent on a set of processes separate from those producing habituation itself (Thompson and Spencer, 1966; Carew et al., 1971).

Behavioral analysis of this decrement clearly suggested that it is not due to an alteration in the cell's contractile mechanisms. The threshold for eliciting contractions using electrical or photic stimuli was the same in control animals and animals habituated to mechanical stimuli (Wood, 1970a, 1973). Stentor also do not habituate to repetitively applied suprathreshold electrical stimuli. Thus, the contractile mechanism of habituated animals appears to be unaltered by the habituation process, suggesting that the locus of change is in the receptor mechanisms.

This conclusion was partially confirmed by electrophysiological results (Wood, 1971). Though microelectrodes were rapidly encapsulated at room temperature, receptor potentials (less than $10 \mathrm{mV}$ ) were still recorded when an impaled animal was mechanically stimulated (Wood, 1970b). Large receptor potentials elicited action potentials that, in turn, triggered ciliary reversal and bodily contractions. During a sequence of 5-11 1/min mechanical stimuli, these receptor potentials were observed to decrease progressively, whereas the action potential amplitude remained unaltered. The final stimulus in each sequence elicited a small receptor potential which failed to elicit an action potential and contraction. Thus, a correlation between habituation of the behavioral response and diminution of the receptor potential was observed, but it was limited because of the instability of the recording site and resultant brevity of the stimulus sequences.

\section{Materials and Methods}

Animals. Stentor coeruleus Ehrenberg of the Stella strain were cultured in high-walled petri dishes in a medium containing $2.5 \mathrm{mM} \mathrm{CaCl}_{2}, 1.0$ $\mathrm{mM} \mathrm{MgSO}_{4}, 1.0 \mathrm{~mm} \mathrm{NaNO}$ and $0.1 \mathrm{~mm} \mathrm{KCl}$ buffered to $\mathrm{pH} 7.6-7.9$ with $0.75 \mathrm{~mm}$ Tris- $\mathrm{HCl}$. Heat-killed wheat grains were added to these cultures to support a population of bacteria and small fagellates and ciliates on which the Stentor could feed.

Electrophysiology. Microelectrode recordings were obtained from 10$50 \mathrm{M} \Omega 0.5 \mathrm{M} \mathrm{KCl}$-filled glass microelectrodes. The voltage electrode was connected via a $\mathrm{Ag} / \mathrm{AgCl}$ half cell to a unity gain preamplifier, 
which was the input to an oscilloscope and an $\mathrm{A} / \mathrm{D}$ converter and microprocessor. The $A / D$ converter functioned at a sampling rate of 1 $\mathrm{KHz}$. Data were taken both from film records of the oscilloscope screen and the microprocessor printout. All voltage records were corrected for the presence of tip potentials. Currents injected through the current electrode were monitored using a virtual ground circuit. Mechanical stimuli were applied by moving the impaling microelectrodes, and hence the impaled animal, $25 \mu \mathrm{m}$ or less through the medium (Wood, 1982). The speed of this movement was monitored by a linear velocity transducer in series with the solenoid armature that produced the movement.

Recordings were obtained from 2 different microelectrode placements. In the first study, animals conlaining a large, $100-150 \mu \mathrm{m}$ vacuole filled with a cannibalized Stentor were selected for testing. The voltage and current microelectrodes were driven simultaneously through the frontal field and, in successful cases, into the vacuole. Microelectrodes were placed in vacuoles to avoid their rapid encapsulation and ejection as normally occurs to cytoplasmic microelectrodes during room temperature recording. In successful penetrations, the vacuole remained fixed relative to the microelectrodes, though other portions of the animal could be moved relative to them. Recordings from these electrodes showed steady-state potentials near $0 \mathrm{mV}$, resistive coupling between the electrodes that was at least 4 times the coupling found between intracellular electrodes, and markedly reduced capacitive coupling. These membrane parameters remained constant for as long as an hour.

In subsequent studies, the microelectrodes were positioned intracellularly by inserting them through the frontal field of animals lacking large vacuoles but chilled to $8.5-10^{\circ} \mathrm{C}$. Only animals with resting potentials exceeding $-48 \mathrm{mV}$, action potentials exceeding $60 \mathrm{mV}$, and input resistances greater than $10 \mathrm{M} \Omega$ were retained for further study. The space constant of Stentor is at least 4 times its diameter (Wood, 1982); therefore, all areas of the cell membrane should have been under good voltage control during the voltage clamp studies.

After an initial determination of membrane properties was made, mechanical stimuli were applied at a rate of 1 every $3 \mathrm{~min}$ until 3 successive receptor potentials or receptor currents showed the same amplitude. Thirty to $45 \mathrm{~min}$ of testing were often required before this asymptotic level was reached. Only animals producing receptor potentials exceeding $5 \mathrm{mV}$ or receptor currents exceeding $2 \mathrm{nA}$ were retained for further study. The stimulus sequences used in the various habituation studies are outlined in Results. The membrane properties of each preparation were redetermined at the end of the experiment; all the reported data were obtained from animals showing no significant changes in membrane properties throughout the recording period.

\section{Results}

\section{Intravacuolar recordings}

By simultaneously placing both current and voltage electrodes into a large food vacuole containing a cannibalized Stentor, longterm recordings of receptor potentials (up to $20 \mathrm{mV}$ ) and action potentials (up to $75 \mathrm{mV}$ ) were obtained and used to generate a correlation between behavior and transmembrane potentials. As in the earlier studies, repetitive mechanical stimulation resulted in a progressive and highly significant $(t(4)=9.12, p<$ $0.001)$ diminution of the amplitude of the recorded receptor potential (Fig. 1). During the course of the $201 / \mathrm{min}$ stimuli used, the probability that a stimulus would elicit an action potential and contraction decreased progressively in a manner highly correlated $(r=0.99)$ with the diminution in the receptor potential amplitude. On the average, animals produced action potentials and contracted in response to only 4 of the last 10 stimuli. The amplitude of these action potentials showed no decrement relative to those elicited at the onset of the stimulus sequence. These data confirm that habituation in Stentor is attributable to an alteration in the mechanism that generates the receptor potential.

\section{Intracellular recordings}

Stimulation required to produce habituation. Stable, long-lasting intracellular recordings can be obtained only when the animals

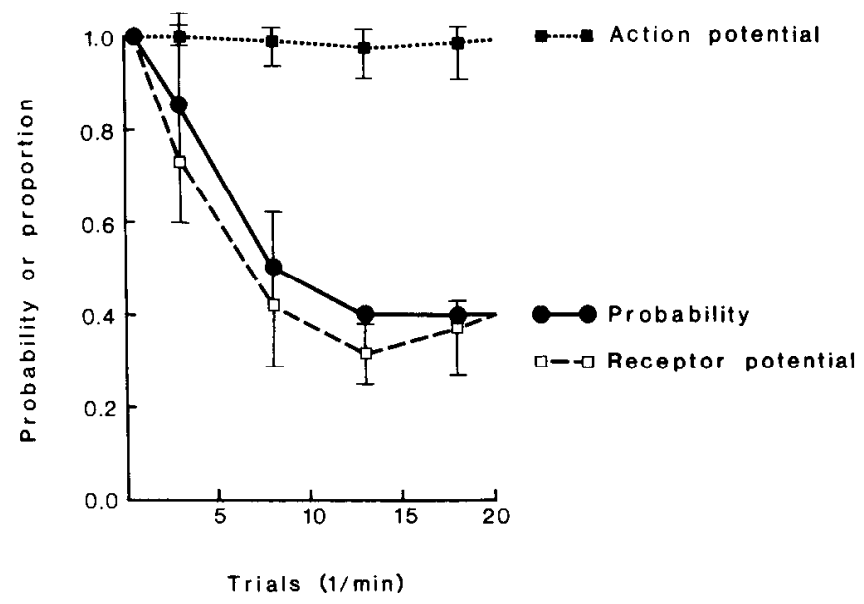

Figure 1. The probability that Stentor will contract during the course of twenty $1 / \mathrm{min}$ mechanical stimuli plotted together with the normalized receptor potential and action potential amplitudes recorded simultaneously from the same animals $(n=5)$. Means \pm SE.

are chilled to between 8.5 and $10^{\circ} \mathrm{C}$ (Wood, 1982). Under these conditions, mechanical stimuli elicit receptor potentials that generally have maximal amplitudes between 5 and $15 \mathrm{mV}$ and typically do not elicit action potentials. Receptor potentials elicited from chilled animals by repetitive $1 /$ min mechanical stimuli decreased in amplitude by a slight and nonsignificant amount $(t(6)=0.71, p>0.05)$ over the course of 20 stimuli (Fig. 2, $A$, $B)$. The difference between these results and those reported in Figure 1 suggests that action potentials, which occurred frequently in the previous study but were not elicited from these chilled animals, are important in the production of habituation. To test this hypothesis, a second group of animals was given 20 $1 / \mathrm{min}$ mechanical stimuli each of which was followed in 200 msec by a depolarizing current pulse sufficient to elicit an action potential. The $200 \mathrm{msec}$ interval was chosen to allow time for receptor potential to decay prior to the onset of the depolarizing current pulse, though this interval is longer than the time between mechanical stimulation and action potential onset as recorded from animals at room temperature. This sequence of combined mechanical and electrical stimulation produced a rapid and pronounced decrease in the amplitude of the receptor potential, which followed a time course similar to that observed in the previous study (Fig. 1). To test if action potential production alone is sufficient to produce this diminution, a third group of animals was given depolarizing current pulses only for $201 / \mathrm{min}$ trials, except on trials 10 and 20 when mechanical stimuli alone were applied. A large decrement in the amplitude of the receptor potential elicited on trial 10 was observed in animals that had received 9 preceding current pulses and had produced 9 action potentials. Two-way analysis of variance revealed a significant interaction between the treatments used and the number of trials $(F(4,36)=7.58, p<0.01)$. Post hoc comparisons showed that the receptor potentials of groups given electrical stimuli alone or combined mechanical and electrical stimuli were significantly depressed on trials 10 and 20 compared to those of groups given mechanical stimuli alone (Scheffé test; $p<0.01$ in all cases). These diminutions were specific to receptor potential amplitude, since resting potentials, membrane resistances, and action potential amplitudes were not significantly altered over the period of stimulation in any of the groups. These diminutions were also transitory since the recep- 
A
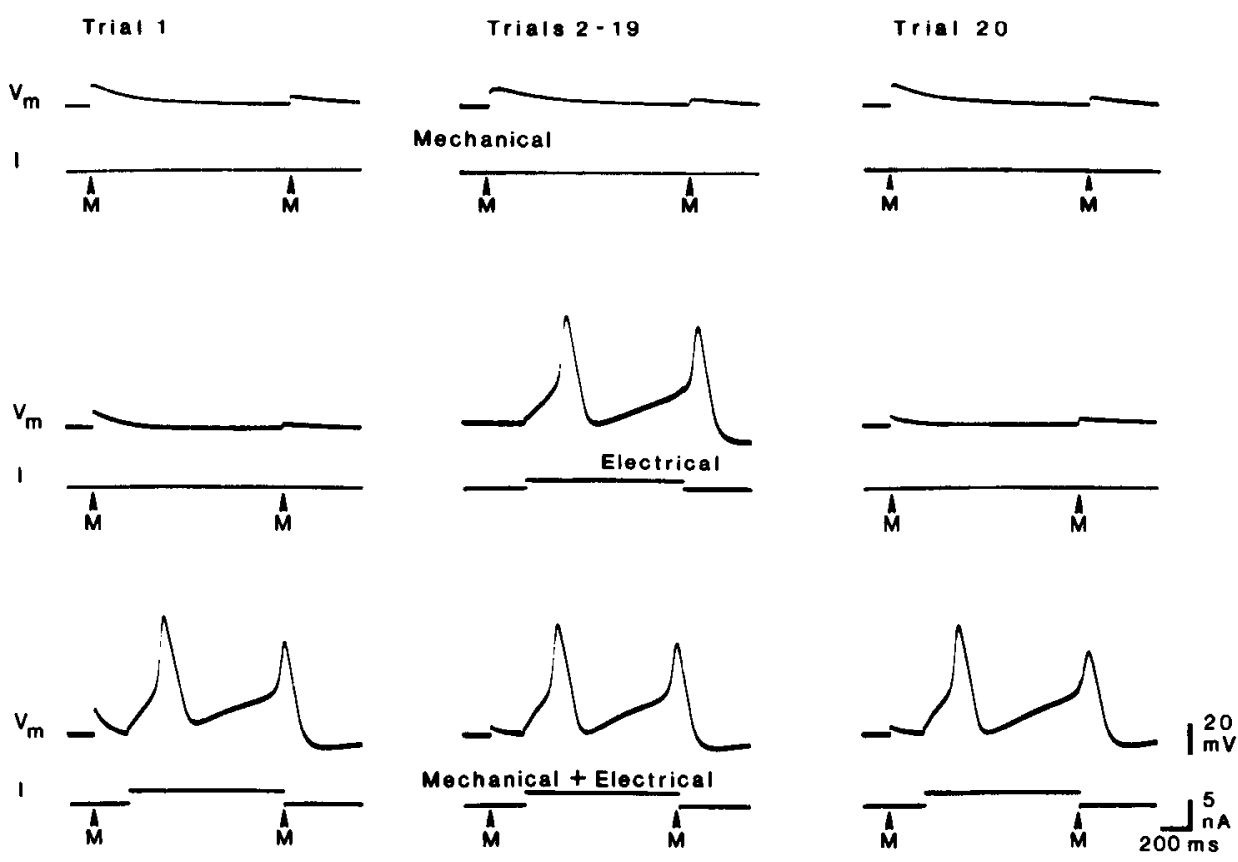

B

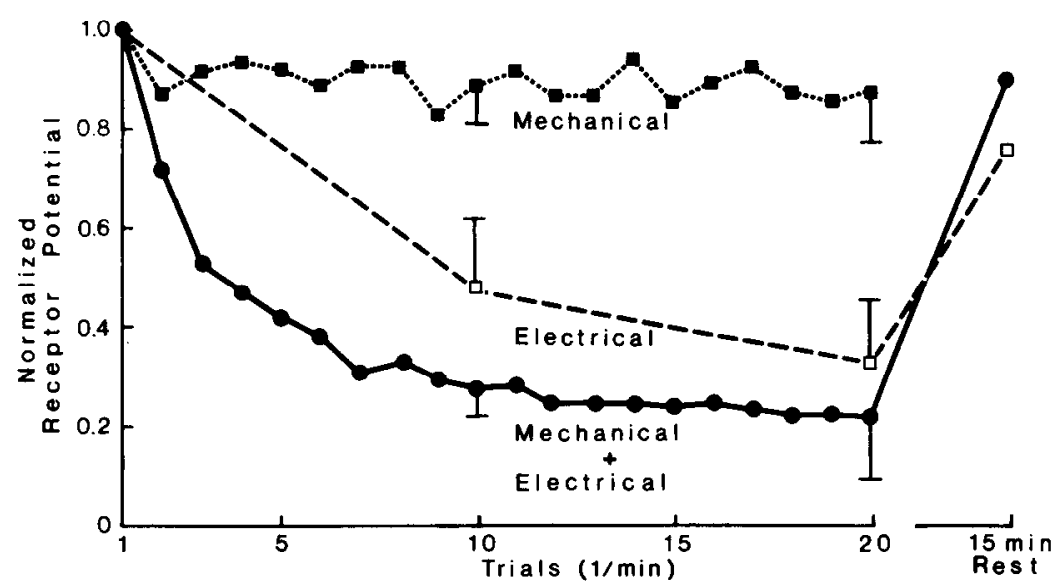

tor potentials in all groups returned to values insignificantly different from prestimulation values following a $15 \mathrm{~min}$ rest period after the end of the stimulation sequence. The greater depression of receptor potential amplitude recorded on trials 10 and 20 from animals given combined mechanical and electrical stimuli as compared to animals receiving electrical stimuli alone did not reach significance (Scheffé test, $p>0.05$ ).

The contribution of mechanical and electrical stimulation to habituation was also tested by mechanically and/or electrically stimulating animals that were under voltage clamp control. The same 3 experimental groups-mechanical stimulation alone, electrical stimulation alone, and combined mechanical and electrical stimulation - were used in these studies, except that a 200 msec voltage step from the $-50 \mathrm{mV}$ holding potential to $0 \mathrm{mV}$ was employed in place of an electrically stimulated action potential. The pattern of results observed in this experiment was almost identical to that observed in the previous study (Fig. 3). The largest decrease in receptor current amplitude occurred in the 2 groups given repetitive voltage steps to simulate action potentials; a planned comparison of the average receptor current on trial 20 recorded from animals given mechanical stimulation alone versus the average receptor currents observed in the 2 electrically stimulated groups was highly significant $(t(36)=$ $3.39, p<0.001)$. As in the previous study, mechanical stimulation alone produced a slight decrease in receptor function, but this decrease was again not significant $(t(4)=1.57, p>0.05)$. However, the planned comparison of trial 10 receptor currents recorded from animals given electrical stimulation alone versus those given combined mechanical and electrical stimulation was significant $(t(36)=1.69, p<0.05)$. A $15 \mathrm{~min}$ rest following the 20 min period of stimulation was sufficient to allow the receptor currents in all groups to return to prestimulation values.

Stimulation during recovery from habituation. In the preceding studies, repetitive mechanical stimulation, when unaccompanied by action potential production, produced only small and nonsignificant diminutions in either receptor potential or recep- 


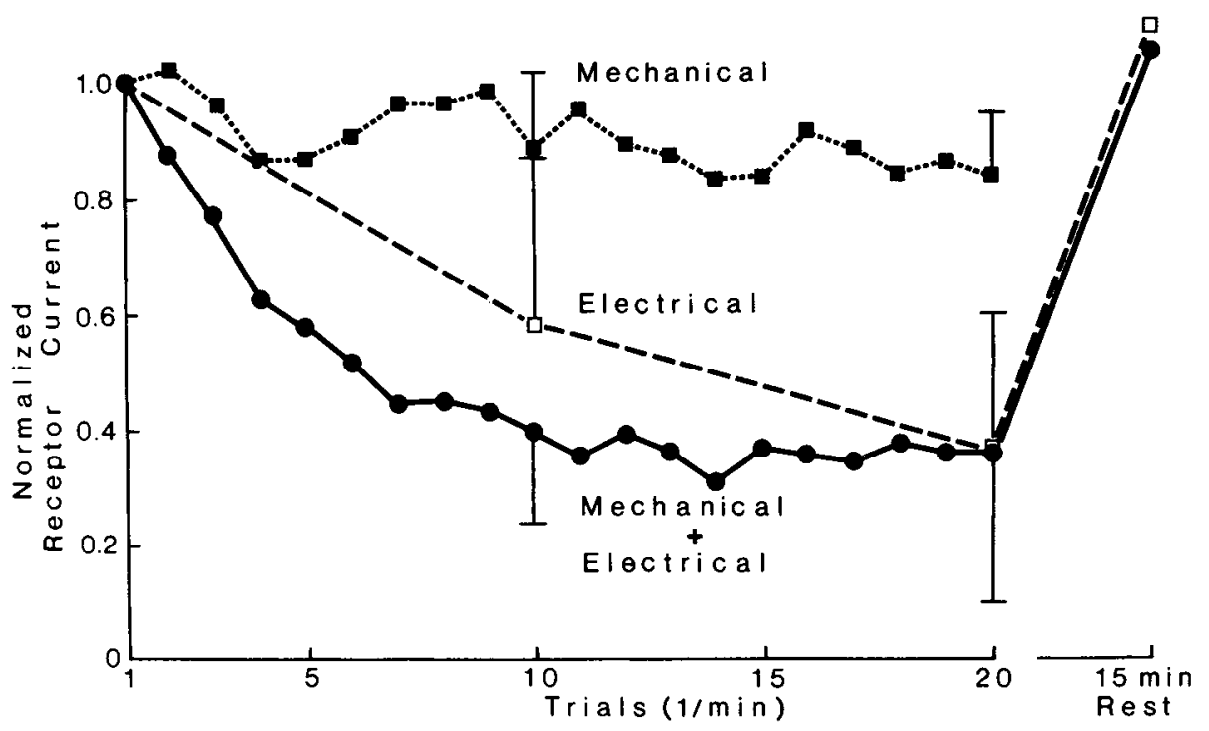

Figure 3. Normalized receptor current amplitudes recorded from voltageclamped cells given mechanical stimulation alone $(n=5)$, depolarizing voltage steps alone (except on mechanical stimulus test trials) $(n=5)$, or combined mechanical stimulation and depolarizing voltage steps $(n=5)$ plotted against trials. Means $\pm \mathrm{SE}$. tor current amplitude. Nevertheless, these small reductions were consistent suggesting that mechanical stimulation or the resultant receptor currents make a small contribution to the habituation process. This possibility was examined further by studying the effect of mechanical stimulation on the process of recovery from habituation. Four groups of animals were initially habituated with 20 trials of combined mechanical and electrical stimulation occurring at a rate of $1 / \mathrm{min}$. Subsequently, animals in the control group were given mechanical stimulation after 10 and $20 \mathrm{~min}$ of recovery time to assess the course of their recovery. Animals in a second group, which had experienced a receptor potential diminution during habituation very similar to that of the control group, were given $1 / \mathrm{min}$ mechanical stimulation throughout the $20 \mathrm{~min}$ recovery period. Receptor potentials recorded from this mechanically stimulated group were significantly $(t(9)=2.03, p<0.025)$ depressed relative to control values after $20 \mathrm{~min}$ of recovery (Fig. 4). A third group was given a suprathreshold electrical stimulus once every $4 \mathrm{~min}$ during the recovery period to determine whether action potential production at this low rate would be sufficient to sustain habituation. This stimulation rate was chosen to mimic the rate at which animals contract at the end of a behavioral test producing habituation. The fourth group received both suprathreshold electrical stimulation once every $4 \mathrm{~min}$ and mechanical stimulation once every minute. This stimulation pattern closely resembles that experienced by animals at the end of the habituation process during behavioral tests. The planned comparison between groups 3 and 4 revealed that the addition of the $1 / \mathrm{min}$ mechanical stimulation to the distributed pattern of electrical stimulation significantly $(t(10)=3.31, p<0.001)$ reduced the recovery of the receptor potential amplitude by 20 min of recovery.

The influence of mechanical stimulation on recovery from habituation was also studied using voltage-clamped cells subjected to the 4 stimulus sequences used above. As in the 2 group comparisons cited above, mechanical stimulation was observed to reduce recovery; however, the groups in this study contained only 2 to 4 animals, so the effects were not statistically significant.

Temporal relation between stimuli. The previous experiments indicate that both mechanical stimulation (or one of its consequences) and action potentials (or one of their consequences) contribute to the process of habituation. This raises the possibility that there is a necessary temporal relation between the 2 events if the maximal degree of habituation is to be attained. To test this possibility, the rate and degree of habituation was studied in 3 groups of animals given $1 / \mathrm{min}$ stimulation scquences with different temporal relationships between the mechanical stimuli and electrically elicited action potentials. In group 1, the mechanical stimulus occurred $200 \mathrm{msec}$ before the onset of the electrical stimulus; in group 2, the mechanical stimulus occurred $600 \mathrm{msec}$ after the offset of the electrical stimulus;

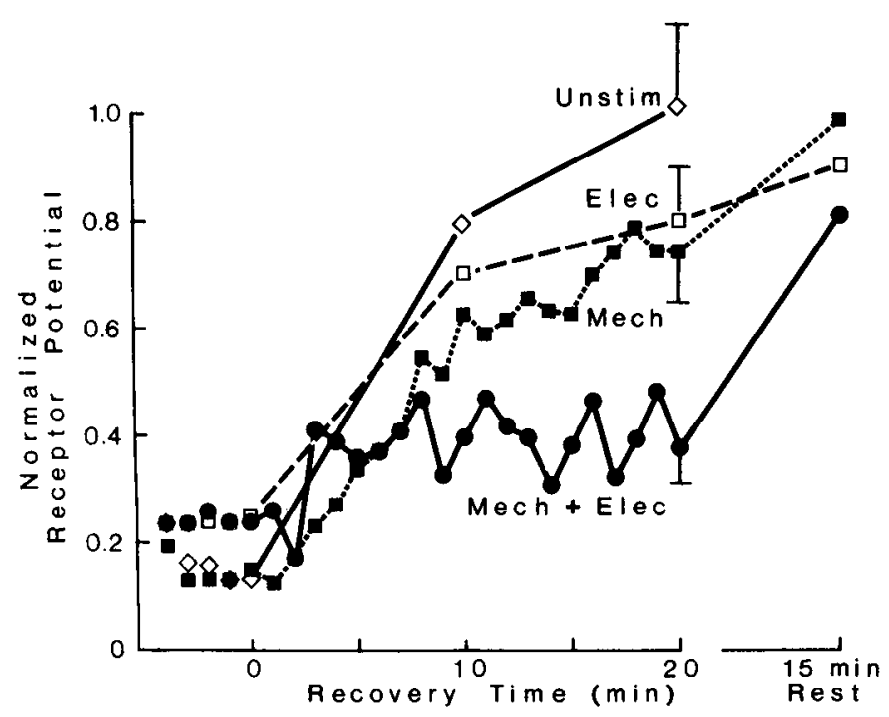

Figure 4. Recovery of normalized receptor potential amplitudes after habituation. All animals were initially given 20 combined mechanical and electrical stimuli to produce habituation of the receptor potential amplitude. The normalized receptor potentials elicited on the last 5 of these 20 trials are plotted as the data points to the left of the origin. During recovery from this habituation, animals were either (1) not stimulated (except for test mechanical stimuli at 10 and $20 \mathrm{~min}$ of recovery) $(n=5)$, (2) given mechanical stimuli every $\min (n=5),(3)$ given depolarizing current pulses once every $4 \mathrm{~min}$ and test mechanical stimuli at 10 and $20 \mathrm{~min}$ of recovery $(n-6)$, or (4) given mechanical stimuli every min and depolarizing current pulses once every $4 \mathrm{~min}(n$ $=5$ ). Means $\pm \mathrm{SE}$. 
Figure 5. Habituation of normalized receptor potential amplitude in groups experiencing different temporal relations between the mechanical and electrical stimuli. Normalized receptor potential amplitudes decayed at similar rates and to similar degrees in groups of animals given either (1) mechanical stimuli, each of which was followed after $200 \mathrm{msec}$ by a depolarizing current pulse $(n=5),(2)$ depolarizing current pulses, each of which was followed in $600 \mathrm{msec}$ by a mechanical stimulus ( $n$ $=5$ ), or (3) mechanical and electrical stimuli spaced $30 \mathrm{sec}$ apart $(n=5)$. After a 15 min rest, animals were given an additional set of 10 mechanical stimuli to test their recovery from habituation. While the recovery of the Elec $\rightarrow$ Mech stimulus group appears greater than that of the other groups, this difference is not significant $(t(10)=1.39$; $p>0.05$ ) and was not maintained on subsequent trials.
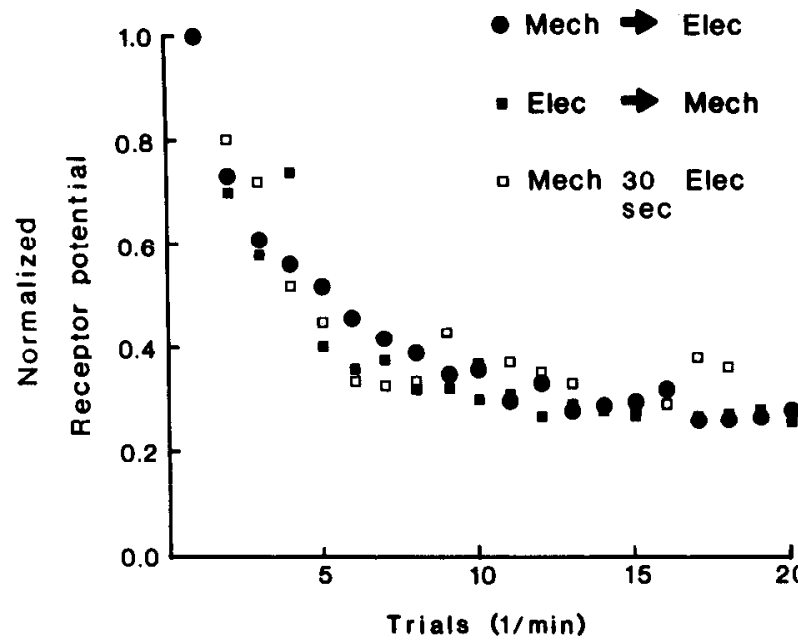

Rest

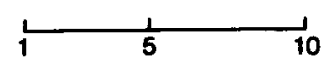

and in group 3, the 2 stimuli were spaced 30 sec apart. The results of this experiment were unequivocal (Fig. 5); all groups exhibited the same rate $(F(38,240)=0.3, p>0.05)$ and degree $(F(2,12)=0.25, p>0.05)$ of receptor potential diminution. Thus, the temporal relation between the 2 stimuli appears inconsequential as a determinant of the habituation process.

\section{Discussion}

In the first study reported above, repetitive mechanical stimulation was observed to produce a progressive diminution in receptor potential amplitude that was highly correlated with the habituation of the animals' contractile response. Action potential amplitude did not change during the repetitive mechanical stimulation. These data confirm that habituation is attributable to a change in receptor function, as had been previously concluded on the basis of behavioral (Wood, 1970a) and preliminary electrophysiological (Wood, 1971) data.

Intracellularly recorded receptor potentials, when unaccompanied by action potentials, underwent only a slight (11\%) diminution during the course of repetitive stimulation. Under voltage-clamp, repetitive elicitation of receptor currents was also found to produce only a slight diminution in their amplitude. Thus, mechanical stimuli and the currents and potentials produced by them are not sufficient to produce the pronounced degree of habituation normally seen during repetitive stimulation of animals. On the other hand, the repetitive elicitation of action potentials by electrical stimulation did produce a marked diminution in reccptor potential amplitude. Under voltageclamp, voltage steps simulating action potentials produced a similar pronounced reduction of receptor currents. Therefore, the pronounced habituation to mechanical stimulation observed in behavioral studies must result from the action potentials triggered by most of the mechanical stimuli applied early in the stimulus sequence. The importance of action potential elicitation is also indicated by behavioral evidence showing that $1 / \mathrm{min}$ suprathreshold electrical stimulation is sufficient to produce considerable habituation to mechanical stimulation (Osborn et al., 1973; D. C. Wood, unpublished observations). However, it remains unclear which of the consequences produced by activation of the voltage-dependent $\mathrm{Ca}^{2+}$ channels is important for habituation-the transmembrane potental changes, transmembrane ionic currents, ciliary reversal, or bodily contractions.

Mechanical stimulation or processes elicited by it also appear to make a minor contribution to the habituation process. In the studies cited above, there are 4 instances in which the receptor potentials or currents of a group receiving repetitive mechanical stimulation alone could be compared with the receptor potentials or currents of a group receiving no stimulation. In all 4 cases, the group receiving mechanical stimulation had smaller receptor potentials or currents, but in only one case was this diminution significant (at the $p<0.025$ level). In an additional 4 instances, the effect of mechanical stimulation combined with some pattern of electrical stimulation could be compared with the effect of the electrical stimulation alone. Again, in all 4 cases the group receiving the additional mechanical stimulation had smaller receptor potentials or currents, and in 3 of these cases the differences were statistically significant. These electrophysiological results agree with data from previous behavioral studies (Wood, 1972) that showed that Stentor triggered by electrical stimuli to produce the same number and temporal pattern of contractions as had been produced by animals habituated with repetitive mechanical stimuli were less habituated than were the mechanically stimulated animals. Taken together, these data indicate that mechanical stimulation makes a contribution to behavioral habituation beyond its effect in eliciting action potentials and contractions.

During the studies of recovery from habituation it was observed that recovery in animals electrically stimulated once every 4 min was approximately equal to that of animals receiving mechanical stimulation once every minute. Thus, mechanical stimulation was only $1 / 4$ as effective as electrical stimulation and action potentials in impeding recovery from habituation. Mechanical stimulation alone appears even less effective relative to action potential production as a means of producing habituation. 
Habituation has been considered a specific form of receptor adaptation both in the particular case of Stentor (Davis, 1974) and more generally (Webb and McBurney, 1971; Catania, 1979). These processes are similar in that both are produced by a decrease in receptor sensitivity. However, receptor adaptation can be observed when only receptor potentials are produced (Fig. 2A); in contrast, pronounced habituation occurs only if action potentials are elicited. Thus, these processes have different cellular mechanisms.

The dependence of habituation on response production is suggestive of the contingent relation between stimulus and response found in some associative learning processes. However, unlike these processes, there is no necessary temporal contiguity required between the mechanical stimulus and the action potential to produce habituation. Furthermore, action potential production alone appears sufficient to produce most of the phenomenon. Thus, habituation does not involve all the contingencies of an associative learned behavior, but its response dependence indicates that a feedback signal from the response to the receptor mechanisms is functionally important in its production.

\section{References}

Carew, T. J., V. Castellucci, and E. R. Kandel (1971) An analysis of dishabituation and sensitization of the gill-withdrawal reflex in Aplysia. Int. J. Neurosci. 2: 79-98.

Castellucci, V., and E. R. Kandel (1974) A quantal analysis of the synaptic depression underlying habituation of the gill-withdrawal reflex in Aplysia. Proc. Natl. Acad. Sci. USA 71: 5004-5008.

Castellucci, V., H. Pinsker, I. Kupfermann, and E. R. Kandel (1970) Neuronal mechanisms of habituation and dishabituation of the gillwithdrawal reflex in Aplysia. Science 167: 1745-1748.

Catania, A. C. (1979) Learning, Prentice-Hall, Englewood Cliffs, NJ.

Davis, W. J. (1974) Plasticity in invertebrates. In Neural Mechanisms of Learning and Memory, M. R. Rosenzweig and E. L. Bennett, eds., pp. 430-462, MIT Press, Cambridge, MA.

Farel, P., G. Glanzman, and R. F. Thompson (1973) Habituation of a monosynaptic response in vertebrate central nervous system: Lateral column-motoneuron pathway in isolated frog spinal cord. J. Neurophysiol. 36: 1117-1130.

Gingrich, K. J., and J. II. Byrne (1985) Simulation of synaptic depression, posttetanic potentiation, and presynaptic facilitation of synaptic potentials from sensory neurons mediating gill-withdrawal reflex in Aplysia. J. Neurophysiol. 53: 652-669.

Glanzman, G., and R. F. Thompson (1980) Alterations in spontaneous miniature potential activity during habituation of a vertebrate monosynaptic pathway. Brain Res. 189: 377-390.

Jennings, H. S. (1906) Behavior of the Lower Organisms, Columbia U. P., New York.

Klein, M., E. Shapiro, and E. R. Kandel (1980) Synaptic plasticity and the modulation of the $\mathrm{Ca}^{2+}$ current. J. Exp. Biol. 89: 117-157.

Osborn, D., H. J. Blair, J. Thomas, and E. M. Eisenstein (1973) The effects of vibratory and electrical stimulation on habituation in the ciliated protozoan, Spirostomum ambiguum. Behav. Biol. 8: 655664.

Spencer, W. A., R. F. Thompson, and D. R. Neilson, Jr. (1966) Decrement of ventral root electronus and intracellularly recorded PSPs produced by iterated cutaneous afferent volleys. J. Neurophysiol. 29: 253-274.

Thompson, R. F., and W. A. Spencer (1966) Habituation: A model phenomenon for the study of neuronal substrates of behavior. Psychol. Rev. 73: 16-43.

Webb, C. H., and D. H. McBurney (1971) Salivary habituation: Quantiative similarities to sensory adaptation. Am. J. Psychol. 84: $501-$ 512.

Wood, D. C. (1970a) Parametric studies of the response decrement produced by mechanical stimuli in the protozoan, Stentor coeruleus. J. Neurobiol. 1: 345-360.

Wood, D. C. (1970b) Electrophysiological studies of the protozoan, Stentor coeruleus. J. Neurobiol. 1: 363-377.

Wood, D. C. (1971) Electrophysiological correlates of the response decrement produced by mechanical stimuli in the protozoan, Stentor coeruleus. J. Neurobiol. 2: 1-11.

Wood, D. C. (1972) Generalization of habituation between different receptor surfaces of Stentor. Physiol. Behav. 9: 161-165.

Wood, D. C. (1973) Stimulus-specific habituation in a protozoan. Physiol. Behav. 11: 349-354.

Wood, D. C. (1982) Membrane permeabilities determining resting, action, and mechanoreceptor potentials in Stentor coeruleus. J. Comp. Physiol. 146: 537-550.

Zucker, R. S. (1972) Crayfish escape behavior and central synapses. II. Physiological mechanisms underlying behavioral habituation. J. Neurophysiol. 35: 621-637. 